\title{
Current and future strategies for the nutritional management of cardiometabolic complications of androgen deprivation therapy for prostate cancer
}

\author{
Lauren Turner, Karen Poole, Sara Faithfull and Bruce A. Griffin* \\ Faculty of Health and Medical Sciences, University of Surrey, Guildford, Surrey GU2 7XH, UK
}

\section{Abstract}

Androgen deprivation therapy (ADT) is used widely as part of a combined modality for the treatment of prostate cancer. However, ADT has also been associated with the development of cardiometabolic complications that can increase mortality from cardiovascular events. There is emerging evidence to suggest that ADT-related cardiometabolic risk can be mitigated by diet and lifestyle modification. While the clinical focus for a nutritional approach for achieving this effect is unclear, it may depend upon the timely assessment and targeting of dietary changes to the specific risk phenotype of the patient. The present review aims to address the metabolic origins of ADT-related cardiometabolic risk, existing evidence for the effects of dietary intervention in modifying this risk, and the priorities for future dietary strategies.

Key words: Prostate cancer: Androgen deprivation therapy: Metabolic syndrome: Cardiometabolic risk: Nutritional management

Introduction: prostate cancer: prevalence, risk factors and treatments

Prostate cancer is the most common cancer in men, affecting $1 \cdot 1$ million men worldwide in 2012 . In the same year, there were 47300 new cases of diagnosed prostate cancer, and 10800 deaths from the disease in the $\mathrm{UK}^{(1)}$. While there are no clear modifiable risk factors for prostate cancer, family history, diet and lifestyle and ageing have all been linked to its development, with $54 \%$ of men diagnosed in the UK being over 70 years of age. Prostate cancer may be classified as an adenocarcinoma of the prostate gland which can progress from a relatively normal, low-grade prostatic intra-epithelial neoplasm to a high-grade metastatic cancer, with metastases occurring typically in the seminal vesicles, bladder and bones of the pelvis, upper legs and lower spine. The progression of prostate cancer is typically slow, earning it the reputation of being a cancer that men die 'with', rather than 'from', but there are subtypes of the disease that are aggressive and develop rapidly. Prostate cancer is also associated with a high rate of recovery, with $84 \%$ of men in the UK surviving for 10 years or longer after diagnosis $^{(2)}$.

The growth and function of the prostate is heavily influenced by androgens, and chiefly testosterone, which plays a key role in promoting tumour growth. Hence, effective anti-cancer therapy for locally advanced disease employs systematic approaches to reduce exposure to testosterone by the surgical removal of the testes that produce $90 \%$ of the hormone (orchiectomy), or by the administration of drugs that suppress the production of testosterone (androgen deprivation therapy; ADT). These approaches are used in combination with localised treatments that include brachytherapy or external beam radiotherapy. ADT includes anti-androgen drugs that act at the level of the testes, and gonadotrophin-releasing hormone (GnRH) agonists and antagonists, which suppress the production of testosterone through the hypothalamic-pituitary-gonadal axis. While ADT is highly effective as a systemic treatment as part of combined modality therapy, and has been proven to reduce cancer-specific mortality, its use, and specifically the use of GnRH agonists, has been associated with the co-morbidity of increased risk of $\mathrm{CVD}^{(3,4)}$. This CVD risk is characterised by increased cardiometabolic abnormalities which have a rapid onset $^{(5)}$ and underlie an increased prevalence of premature cardiac events such as myocardial infarction, chronic heart failure and stroke in prostate cancer patients within 6 months after ADT has commenced ${ }^{(6-8)}$. While clinical recognition of an association between ADT and increased risk of cardiac events comes in the form of contraindications on ADT drug labels, and the United States Food and Drug Administration's guidelines for the management and targeting of ADT in prostate cancer patients in clinical practice ${ }^{(9)}$, evidence for a causal relationship between ADT and CVD mortality remains contentious. Reasons for this include confounding of the evidence by the advanced age of patients, cross-cultural differences in response to ADT, and general lack of data from randomised controlled trials

Abbreviations: ADT, androgen deprivation therapy; GI, glycaemic index; GnRH, gonadotrophin-releasing hormone; MetS, metabolic syndrome; NAFLD, nonalcoholic fatty liver disease; PREDIMED, Prevención con Dieta Mediterránea; RCT, randomised controlled trial; SAT, subcutaneous adipose tissue, VAT, visceral adipose tissue.

* Corresponding author: Professor Bruce A. Griffin, email b.griffin@surrey.ac.uk 
(RCT), all of which have increased uncertainty as how best to reverse ADT-related cardiometabolic risk. This review examines the origins and underlying pathophysiology of ADT-related cardiometabolic risk, and asks whether existing definitions of the metabolic syndrome (MetS) under-report cardiometabolic risk for men with prostate cancer, and if this risk could be mitigated through dietary intervention.

\section{Evidence that androgen deprivation therapy increases CVD} morbidity and mortality; explanations for the inconsistency between observational studies and randomised controlled trials

Keating et al. $^{(10)}$ and others ${ }^{(11)}$ were amongst the first to show that ADT, and specifically GnRH agonists, increased risk of incident diabetes and CVD (adjusted hazard ratios 1.44 and $1 \cdot 16$, respectively), and the cardiac events of myocardial infarction and sudden cardiac death (adjusted hazard ratios 1.11 and $1 \cdot 16$, respectively). These findings were soon corroborated by further primary studies, and a highly publicised metaanalysis of observational prospective cohort studies that showed GnRH agonists to be associated, unequivocally, with an increased risk of fatal and non-fatal CVD ${ }^{(12)}$. These associations have been linked to the adverse effects of ADT on body composition, insulin sensitivity and other cardiometabolic risk factors described below ${ }^{(6)}$, and also to the specific effects of GnRH agonists in precipitating cardiac events by promoting vascular dysfunction ${ }^{(13-15)}$ and destabilising atherosclerotic plaque $^{(16)}$. Nevertheless, RCT have generally failed to substantiate a direct, and thus causal, link between ADT/GnRH agonists and cardiac mortality ${ }^{(17-21)}$ (Fig. 1). One meta-analysis of RCT identified several possible confounding factors that could have contributed to its negative outcome ${ }^{(19)}$. These factors included failure to record early cardiac events that occur characteristically within 6 to 24 months after ADT has commenced in a 10-year follow-up period, the misdiagnoses of cardiac death as a clinical endpoint, and the recruitment of men onto RCT who are healthier than their counterparts in observational studies. The latter is of special importance because the incidence of ADT-induced cardiovascular mortality has been shown to be significantly higher in men with pre-existing CVD and/or history of CVD events ${ }^{(12,22-24)}$. This finding does not exclude men without a previous history of CVD, in which GnRH agonists are also associated with premature cardiac events ${ }^{(25)}$, but has led to a consensus of opinion that all future RCT should stratify patients according to cardiac co-morbidity at baseline. It is also noteworthy that observational, prospective cohort studies produce evidence for associations between external factors or treatments and disease outcomes, but not evidence for causality which requires a higher level of evidence, from RCT.

How do the origins and characteristics of androgen deprivation therapy-related cardiometabolic risk differ from classic metabolic syndrome?

\section{Classic metabolic syndrome}

Cardiometabolic risk can be defined as a constellation of metabolic risk factors that increase the risk of developing and dying prematurely from $\mathrm{CVD}^{(26)}$. These factors provide the defining criteria for the MetS, which originates primarily from an imbalance between energy intake and expenditure that promotes the accumulation and long-term storage of excess body fat in subcutaneous adipose tissue (SAT) and visceral adipose tissue (VAT) and ectopic sites. This increased adiposity also provides an aetiological link between the MetS and many cancers $^{(27,28)}$, which explains the high prevalence of the MetS in many cancer survivors ${ }^{(29)}$.

The various components of the MetS are expressed in a heterogeneous fashion between individuals, and for this reason the syndrome has many definitions, which vary according to the emphasis placed on one or more components ${ }^{(30)}$. All definitions consist of at least five principal components, three of which should be expressed to qualify as having the MetS. These are central adiposity (increased waist circumference), mild hypertension, hyperglycaemia, dyslipidaemia and insulin resistance. While vascular dysfunction, as marked by a loss of endothelial vasodilation capacity and elasticity or stiffening of larger vessels, is not one of the principal components, it has been described as an integrated marker of cardiometabolic risk and early prerequisite of atherosclerosis that develops under the combined influence of other risk factors. The expression of abnormalities in glucose and lipid metabolism is particularly marked in the postprandial phase. This applies especially to the impaired removal of plasma TAG, the time course of which can take over $6 \mathrm{~h}$ after a fat-containing meal ${ }^{(31)}$. This extended time interval overlaps naturally with the frequency of food intake and absorption of dietary fat, placing most healthy individuals in a postprandial state to some degree throughout the day and during sleep.

There are marked ethnic and cultural differences in how the type and distribution of body fat contributes to the development and expression of cardiometabolic risk. The accumulation of deep, VAT is a major prerequisite for the development of cardiometabolic risk and diabetes in white Caucasians and South Asians, and other ethnic groups who adopt and adapt to a Westernised diet and inactive lifestyle ${ }^{(32)}$. Possible explanations for why VAT should be so closely linked to cardiometabolic risk are that adipocytes in VAT express greater insulin resistance and higher rates of intracellular lipolysis that elevates serum levels of NEFA that can promote further insulin resistance. VAT is also in close proximity to key metabolic organs like the liver and pancreas, and produces adipokines that confer a proinflammatory phenotype on this type of adipose tissue ${ }^{(33)}$. In contrast, the anatomically more superficial SAT is believed to have a role in acting as a reservoir or 'fat sink' to protect peripheral tissues and the viscera from being overloaded with $\mathrm{fat}^{(34)}$. As such, the variable capacity of SAT to either retain or release fat may be a major determinant of preventing or promoting the accumulation of visceral and ectopic fat, respectively. Interestingly, these determinants of classic MetS may not be the same progenitors of ADT-related cardiometabolic risk.

\section{Androgen deprivation therapy-related cardiometabolic risk}

ADT-related cardiometabolic risk shares several of the components of classic MetS - insulin resistance, dyslipidaemia, mild 
(a)

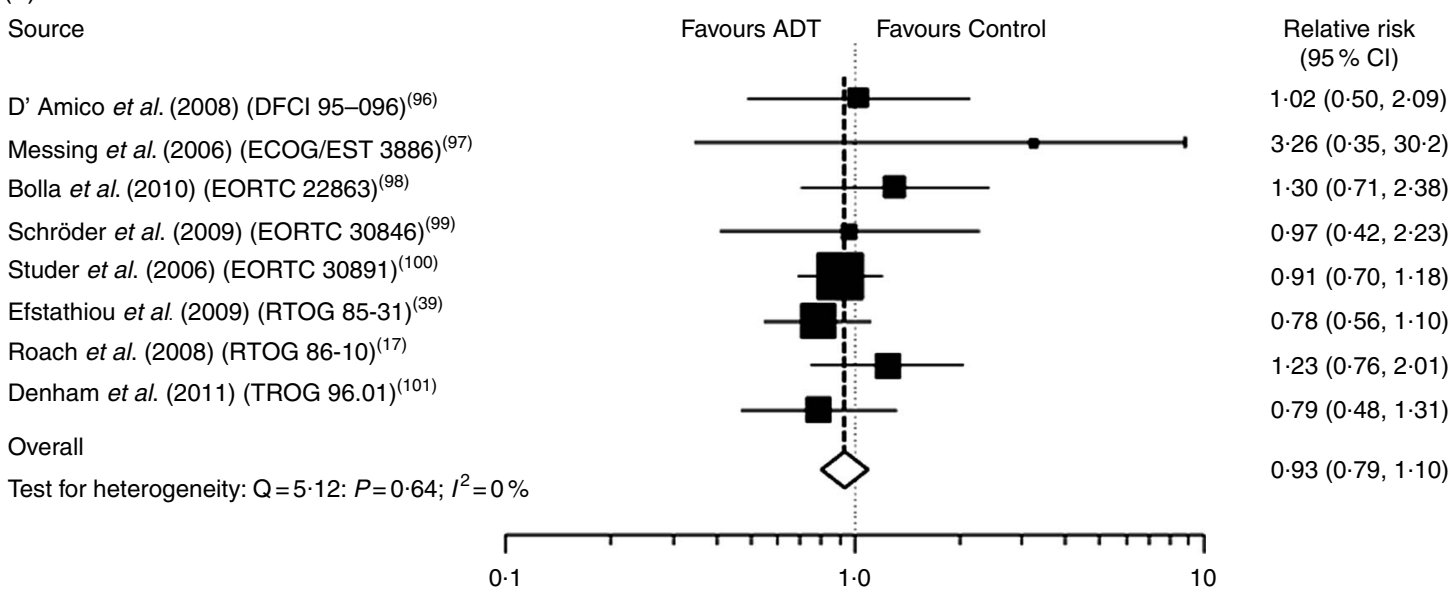

(b)

Azoulay et al. (2011) (stroke) ${ }^{(102)}$

Hu et al. (2012) (pulmonary embolism) ${ }^{(103)}$

Hu et al. (2012) (peripheral arterial disease) ${ }^{(103)}$

Jesperson et al. (2014) (MI) ${ }^{(6)}$

Jesperson et al. (2014) $\left(\right.$ stroke) ${ }^{(6)}$

Keating et al. (2006)-SEER (CHD) ${ }^{(10)}$

Keating et al. (2006)-SEER (MI) ${ }^{(10)}$

Keating et al. (2010)-Veterans Health Administration (CHD) ${ }^{(104)}$

Keating et al. (2010)-Veterans Health Administration (MI) ${ }^{(104)}$

Keating et al. (2010)-Veterans Health Administration (stroke) ${ }^{(104)}$

Martin-Merino et al. (2011) (MI) $)^{(105)}$

Van Hemelrijck et al. (DVT) $)^{(106)}$

Van Hemelrijck et al. (2010) (pulmonary embolism) ${ }^{(106)}$

Van Hemelrijck et al. (2010) (arrhythmia) ${ }^{(107)}$

Van Hemelrijck et al. (2010) (IHD) ${ }^{(107)}$

Van Hemelrijck et al. (2010) (non-fatal stroke) ${ }^{(107)}$

Overall $\left(I^{2}=84.7 \% P<0.001\right)$
$1 \cdot 17(1.01,1.34) \quad 6.35$

$1.29(1 \cdot 18,1 \cdot 40) \quad 7.56$

$1.45(1.37,1.54) \quad 8.05$

$1.28(1.03,1.58) \quad 4.70$

$1.25(1.01,1.54) \quad 4.86$

$1 \cdot 18(1 \cdot 11,1 \cdot 25) \quad 8.06$

$1.24(1.08,1.42) \quad 6.40$

$1.59(1.43,1.77) \quad 7.13$

$1.69(1.37,2.09) \quad 4.77$

$1.65(1.39,1.96) \quad 5.62$

$1.34(0.99,1.82) \quad 3.20$

$2 \cdot 17(1 \cdot 79,2 \cdot 64) \quad 5 \cdot 10$

$1 \cdot 21(0.99,1 \cdot 48) \quad 5 \cdot 01$

$1 \cdot 16(1.06,1 \cdot 27) \quad 7.42$

$1.45(1.36,1.54) \quad 7.93$

$1.47(1.37,1.58) \quad 7.80$

$1.38(1.29,1.48) \quad 100.00$

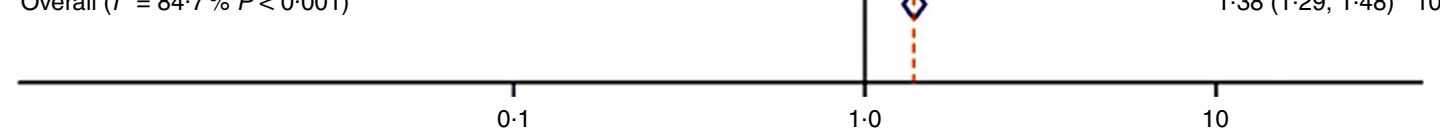

Relative risk (95\% Cl)

Fig. 1. Androgen deprivation therapy (ADT) and relative risk of CVD mortality in meta-analyses of randomised controlled trials (a) and prospective cohort studies (b). Data taken from Nguyen et al. ${ }^{(19)}$ and Bosco et al. ${ }^{(12)}$. Values on the far right in (b) are study weights in \%. DFCI, Dana-Farber Cancer Institute; ECOG, Eastern Cooperative Oncology Group; EORTC, European Organisation for Research and Treatment of Cancer; RTOG, Radiation Therapy Oncology Group; TROG, Trans Tasman Radiation Oncology Group; MI, myocardial infarction; SEER, Surveillance, Epidemiology, and End Results; DVT, deep vein thrombosis.

hypertension and vascular dysfunction ${ }^{(13,35)}$ - but is also distinct from the latter as it develops more rapidly from a single metabolic cause, which is the deficiency or relative absence of testosterone. The pathophysiological effects of testosterone deficiency are of fundamental importance to the unique expression of ADT-induced cardiometabolic risk. At a molecular level, testosterone deficiency alters the pattern of cell differentiation, so that pluripotent stem cells develop into subcutaneous adipocytes rather than skeletal muscle myocytes ${ }^{(36)}$. This effect underlies the earliest adverse effect of ADT on body composition, which is characterised by a pronounced loss of skeletal muscle (sarcopenia) ${ }^{(37)}$ and accumulation of body fat. The latter is marked by the specific accumulation of SAT within 3 months of commencing ADT, as distinct from VAT in classic
$\mathrm{MetS}^{(38)}$ (Fig. 2). This has been followed within 6-12 months after commencing ADT in cohort studies by the development of cardiometabolic risk factors and significant increase in the onset of premature cardiac events (for example, myocardial infarction, stroke) in some $e^{(6-8)}$, but not other studies ${ }^{(17,18,21,39,40)}$. One notable feature of ADT that differs from classic MetS is the preservation or even elevation of serum HDL. However, the high incidence of premature cardiac events on ADT suggests that any cardioprotective effects of HDL may be offset by the adverse effects of ADT on other CVD risk factors. Evidence for the effects of ADT on vascular function in humans has been mixed, with reports of increased arterial stiffness and loss of endothelial cell function in some studies ${ }^{(13-15)}$, but not in others $^{(35,41)}$. There is evidence, exclusively from animal models, 

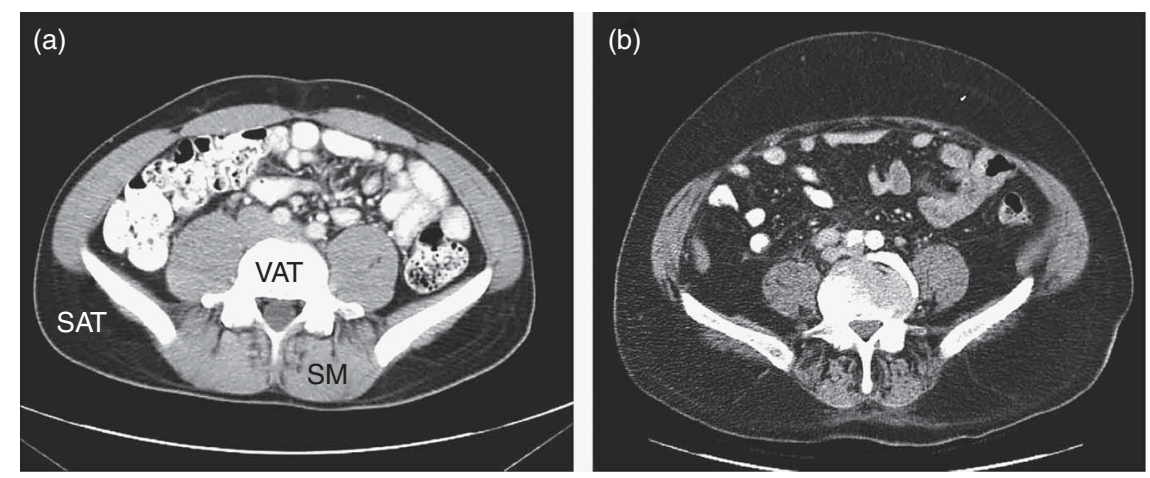

Fig. 2. Abdominal MRI before (a) and after (b) androgen deprivation therapy (ADT) ${ }^{(38)}$, showing the accumulation of subcutaneous adipose tissue (SAT), and relative depletion of visceral adipose tissue (VAT) and loss of skeletal muscle (SM) after ADT.

Table 1. Physical and biochemical characteristics associated with classic metabolic syndrome and androgen deprivation therapy (ADT)-related cardiometabolic risk

\begin{tabular}{ll}
\hline Classic metabolic syndrome & ADT-related cardiometabolic risk \\
\hline Mild hypertension & Mild hypertension \\
Dyslipidaemia & Dyslipidaemia \\
Insulin resistance & Insulin resistance \\
Vascular dysfunction & Vascular dysfunction \\
Accumulation of visceral fat & Accumulation of subcutaneous \\
Central adiposity (increased waist & adipose tissue \\
circumference) & Rapidly develops from a single \\
Hyperglycaemia & metabolic cause \\
Abnormalities in glucose and lipid & Pronounced loss of skeletal muscle \\
metabolism (impaired clearance & (sarcopenia) \\
of plasma TAG) & Preservation or elevation of \\
& serum HDL \\
\hline
\end{tabular}

that acute ADT promotes inflammation, atherosclerosis and destabilises plaque ${ }^{(16)}$, and that this relates specifically to GnRH agonists and not antagonists. A comparison between the components of classic MetS and ADT-induced cardiometabolic risk factors is shown in Table 1.

\section{Relevance of sarcopenia and sarcopenic obesity to cardiometabolic risk}

The age-related decline in skeletal muscle mass and function is defined as sarcopenia, which when superimposed on obesity gives rise to sarcopenic obesity. Sarcopenia and sarcopenic obesity have both been related to insulin resistance and dysglycaemia $^{(42)}$, and more recently to a higher risk of the MetS in a Korean cohort ${ }^{(43)}$, and are common findings in response to $\mathrm{ADT}^{(44)}$. From a mechanistic standpoint, insulin resistance is believed to produce a metabolic milieu that imbalances the anabolism and catabolism of skeletal muscle protein and leads to sarcopenia. The subsequent loss of skeletal muscle then reduces the capacity to store glucose as glycogen, and to a lesser extent TAG, but also to a decline in the metabolic capacity to oxidise glucose and fatty acids that will ultimately promote fat storage. These metabolic events have the potential to fuel a vicious cycle that increases cardiometabolic risk in sarcopenic obesity to a greater extent than can be found in either condition alone. Moreover, ageing will also contribute to the risk of developing sarcopenia and cardiometabolic risk through the natural decline of testosterone production, as was evident in a prospective study from the expression of hyperglycaemia and type 2 diabetes at 10, 20 and 30 years before the development of sarcopenia ${ }^{(45)}$.

\section{Importance of ectopic fat and partitioning of fat between} subcutaneous adipose tissue and visceral adipose tissue

When alcohol can be excluded as a cause of fat accumulation in the liver, and liver fat exceeds $5 \%$ by weight, this condition is known as non-alcoholic fatty liver disease (NAFLD). Fatty liver disease (hepatic steatosis) was originally believed to be clinically benign, but has emerged more recently as a critical determinant of cardiometabolic risk, and prerequisite for the development of non-alcoholic steatohepatitis (NASH), a more serious inflammatory condition which can progress to end-stage liver diseases ${ }^{(46)}$. NAFLD has been associated with all of the components of classic MetS. It has been shown to be responsive to diet and lifestyle modification ${ }^{(47)}$, but its expression in ADTrelated cardiometabolic risk is unknown. While the overflow of stored fat from SAT ('fat-sink' theory) provides one possible origin of fat for VAT and ectopic sites such as the liver, the extent to which fatty acids from the expanded SAT depot in ADT may promote fat deposition in the liver and NAFLD is equally unknown. It is possible that the extent of fat accumulation in the liver could contribute to the variability in the severity of ADT-related cardiometabolic risk expression between different individuals (Fig. 3).

Does the metabolic syndrome have clinical utility in the identification and management of androgen deprivation therapy-related cardiometabolic risk?

CVD risk is known to increase with the number of components of the MetS ${ }^{(26)}$, but it has been argued that classic MetS has no diagnostic or prognostic value, since its individual components are equally or more effective in predicting CVD risk than combinations of these components ${ }^{(48)}$. Individual cardiometabolic risk factors are also treated clinically in isolation of one another by drug therapies. Clear differences between ADT and 


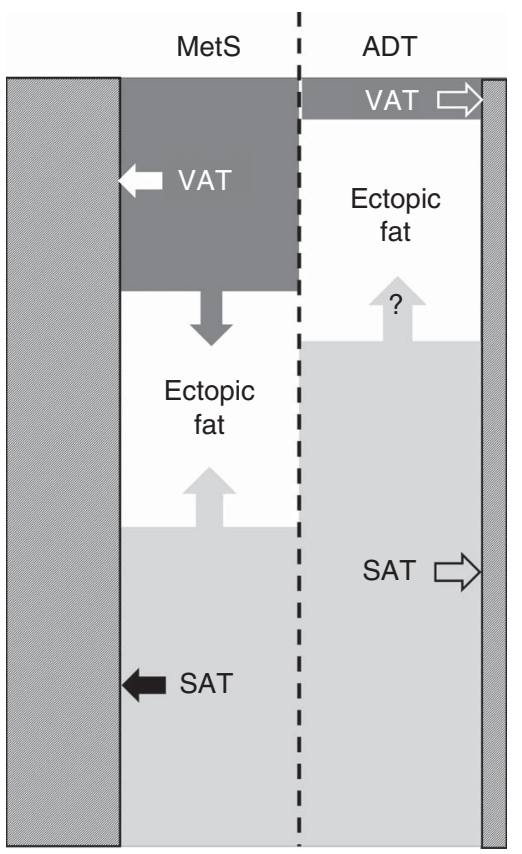

Fig. 3. Relative proportions of stored body fat in visceral adipose tissue (VAT) and subcutaneous adipose tissue (SAT), and potential partitioning (arrows) of stored body fat between VAT, SAT, ectopic sites (for example, liver) and skeletal muscle (//I) in classic metabolic syndrome (MetS) and androgen deprivation therapy (ADT).

classic MetS suggest that the latter may have even less clinical utility in the identification and management of ADT-related cardiometabolic risk. Nevertheless, cardiometabolic risk factors represent sensitive and specific biomarkers of ectopic fat deposition $^{(49)}$, which is an important aetiological factor for the development of CVD, diabetes and chronic liver disease. Also, if cardiometabolic risk can be identified at an early, subclinical stage, this will increase its amenability and responsiveness to diet and lifestyle modification than at a later stage, when longterm drug therapies must be invoked.

By comparison with the lifestyle interventions of weight loss and increased physical activity that are advocated after prostate cancer treatment in cancer survivors, we have relatively little evidence to support the clinical efficacy of dietary interventions.

\section{Search strategy and selection criteria for review}

A systematic search of published literature was conducted to retrieve studies that reported on nutrition interventions (according to WHO definition) or detailed dietary intake after the diagnosis of prostate cancer, as well as reported cardiometabolic risk factors and/or sarcopenia. No restriction was placed on study design or duration of follow-up. Only studies reported in English were included from the following electronic databases: the Cochrane Library, PubMed, MEDLINE, EMBASE and CINAHL. The Index/MeSH (Medical Subject Heading) 'prostate cancer' was combined with terms associated with nutrition interventions and cardiometabolic risk factors (metabolic syndrome, body fat distribution, body mass index, abdominal obesity, morbid obesity, waist circumference, skinfold thickness, waist-hip ratio, hypertension, dyslipidaemia, insulin resistance, hyperglycaemia, blood pressure, cholesterol, diabetes, dysglycaemia, sarcopenia). Additional search strings were constructed using key word terms. All searches were conducted between February and March 2016 and retrieved 1593 published articles since 1 January 2000.

\section{Future strategies for the dietary treatment of androgen deprivation therapy-induced cardiometabolic risk}

Despite similarities between cardiometabolic risk in classic MetS and that induced by ADT, the rapid onset, and distinct and highly variable expression of characteristics in the latter, necessitates a unique and personalised dietary approach. This will require application of the evidence-based effects of diet on ADT-related cardiometabolic risk. However, diet and lifestyle strategies to treat metabolic complications of ADT have predominantly focused on the role of exercise in preference to diet. When the role of diet has been addressed, the quality and detail of information on the interventions have ranged from general healthy eating advice, to more prescriptive guidelines based on the dietary modification of cardiovascular risk, using for example the Adult Treatment Panel III (ATP-III) dietary guidelines ${ }^{(50)}$. The outcome of dietary interventions reported to date has often been poorly described and multi-modal, making it difficult to compare and assimilate results for the design of future dietary interventions (Table 2). In the absence of such data, it would seem prudent to adopt an approach based on the existing evidence for the efficacy of diet on cardiometabolic risk factors in classic MetS. This approach could then be coupled with the concept of personalised nutrition, which is based on the premise that greater improvements in cardiometabolic health can be achieved by the tailoring of dietary modifications to the cardiometabolic risk profile of an individual or, more realistically, group of individuals. In this respect, the coexistence of classic obesity or metabolic obesity, with or without sarcopenia, represent key phenotypic differences for the formulation of an individualised dietary prescription to manage and reduce ADT-related cardiometabolic risk. This approach would provide a more consistent, and evidence-based strategy than many current practices.

\section{Current evidence for the nutritional management of cardiometabolic risk factors}

It is important to emphasise that the nutritional management of cardiometabolic risk does not strictly include the treatment of raised serum LDL-cholesterol. While the latter is an established risk factor for CVD that is associated with increased body weight, obesity and ADT, and is often classified as a cardiometabolic risk factor, its metabolic origins are distinct from the classic cardiometabolic risk factors described above. With the exception of the shared benefits of weight loss, the nutritional management of elevated serum LDL-cholesterol is also distinct from the management of cardiometabolic risk, and delivered via application of the well-documented Portfolio Diet ${ }^{(51,52)}$, which includes weight loss, if appropriate, the replacement of SFA, 


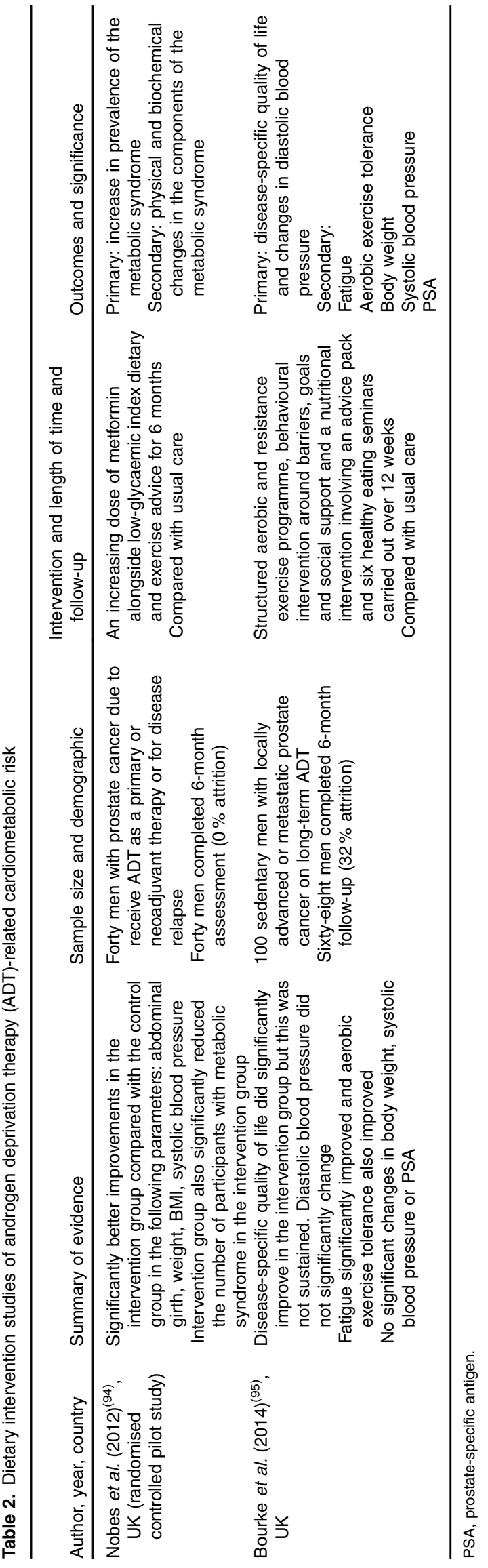

plant sterols, soya protein, viscous fibres and nuts alongside regular clinic visits. This combined dietary approach has been shown to lower LDL-cholesterol more effectively than a low-saturated fat diet alone ${ }^{(52)}$.

In general, diets that restrict the intake of food energy to achieve weight loss have proven efficacy in improving cardiometabolic risk factors in overweight and obese individuals, primarily by reducing total body fat ${ }^{(53,54)}$. Because body composition is difficult to assess without high-resolution imaging techniques, anthropometric measures of BMI, waist circumference and waist:hip ratio are often used as proxies. Prescriptive targets for weight reduction include a sufficient weight loss to achieve a BMI of $25 \mathrm{~kg} / \mathrm{m}^{2}$ for the improvement of hypertension ${ }^{(55)}$, and a BMI of between 21 and $23 \mathrm{~kg} / \mathrm{m}^{2}$ for reducing prostate cancer aggression ${ }^{(56)}$ and an energy restriction of $600 \mathrm{kcal}(2500 \mathrm{~kJ})$ per $\mathrm{d}$ for the management of obesity ${ }^{(57)}$. Energy restriction can be achieved by the manipulation of portion size and/or energy density of food, and by altering behaviour in ways that suppress appetite, hunger and promote satiety, as for example energy loading early in the day ${ }^{(58)}$, maintaining an adequate quantity and quality of sleep $^{(59)}$, by fasting ${ }^{(60)}$ and through meal replacement ${ }^{(61)}$. Humans have adapted to a pattern of dietary intake in developed societies that provides ready and regular access to high-energy-dense foods, which over time will inevitably contribute to weight gain and obesity. This pattern of food intake is in complete discord with our innate physiology that is designed to withstand prolonged periods of fasting in an environment where food is frequently unavailable ${ }^{(62)}$. The unavoidable imposition of time-restricted feeding or intermittent fasting in an environment where food is not readily available, but weight gain and obesity are rare, provides persuasive evidence for the potential of fasting to improve cardiometabolic health. While there is, as yet, no convincing evidence to show that timerestricted feeding or intermittent fasting is more effective than constant energy restriction in promoting weight loss, there are data to suggest the former may confer additional benefits in terms of reducing cardiometabolic risk factors, above and beyond its impact on weight loss ${ }^{(63,64)}$. In a randomised trial, the timing of food intake was shown to make an impact upon cardiometabolic risk factors in young women, in whom insulin resistance and fasting insulin were significantly better in those who undertook an intermittent energy-restricted dietary pattern, compared with those who continually restricted dietary intake $^{(64)}$. Moreover, various fasting practices have been associated with increased dietary compliance, presumably as a favoured option for those unwilling to restrict food intake on a daily basis ${ }^{(65)}$.

For individuals who express increased cardiometabolic risk, but who are neither overweight nor obese, a condition often referred to as 'metabolic obesity', restricting food energy would be inappropriate and unethical. There is convincing evidence to suggest that the origin of cardiometabolic risk in metabolic and classic obesity arises from increased VAT and excess ectopic fat in key metabolic tissues, such as the liver, pancreas, heart and skeletal muscle ${ }^{(66)}$. There are also reports that these depots of VAT and ectopic fat are reducible by energy-restricted weight loss and by manipulating the quality of dietary macronutrients. 
The latter is addressed in a review by Abete et al. ${ }^{(61)}$ that highlights the importance of personalising diets with a specific macronutrient ratio (30\% protein, $40 \%$ low-glycaemic index (GI) carbohydrates, high amounts of MUFA and $n-3$ fatty acids) as well as fibre, isoflavones, $\mathrm{Ca}$ and antioxidants).

\section{Dietary fat}

While much of the emphasis of replacing SFA with PUFA and/or MUFA is rightfully focused on reducing serum LDL, the exchange of dietary SFA for PUFA also produces favourable effects in lowering VAT and liver fat, and on vascular function $^{(67)}$. In contrast to the established effects of weight loss and physical exercise on increasing insulin sensitivity, there is no definitive evidence to show that isoenergetic changes in diet produce the same effect. While this applies specifically to the replacement of SFA with unsaturated fatty acids ${ }^{(68)}$, a reduction in VAT following the replacement of SFA with unsaturated fatty acids would be expected to reduce insulin resistance in this tissue. Similarly, although the increased intake of long-chain $n$-3 fatty acids in supplemental doses (1-3g/d) from purified marine sources has no impact on insulin sensitivity, these fatty acids exert favourable effects on cardiometabolic risk by reducing serum TAG in the postprandial and post-absorptive phases, improving vascular function, and lowering biomarkers of lowgrade inflammation and blood pressure ${ }^{(69)}$. The cell membranes of hepatocytes taken from patients with NAFLD have also been shown to be relatively deficient in long-chain $n-3$ PUFA and, conversely, rich in $\mathrm{SFA}^{(70)}$. Interestingly, supplements of longchain $n$-3 PUFA are effective in reducing liver fat in NAFLD ${ }^{(71)}$ which may explain, in part, how these fatty acids produce beneficial effects on cardiometabolic risk factors.

\section{Dietary carbohydrate}

Three ways in which dietary carbohydrates can affect cardiometabolic risk factors are via their glycaemic properties, content of free sugars, and indigestible dietary fibre. GI describes the rate and extent at which carbohydrate-rich foods raise serum glucose, and concomitantly insulin, relative to a pure glucose control. High-GI foods have been linked to obesity and increased cardiometabolic risk ${ }^{(72)}$. Although there have been various attempts to list GI on food products, GI is determined by numerous physiochemical factors that complicate its measurement and interpretation, and have confounded its practical application in human dietetics and nutrition. Free sugars, formally known as non-milk extrinsic sugars, are sugars added to sweeten foods and include sucrose, a disaccharide of the monosaccharides glucose and fructose, and fructose. Free sugars have been shown to make a significant contribution to energy intake in populations, and are thus implicated in the development of obesity ${ }^{(73)}$, though other more recent data indicate that dietary fat over a mean range of intakes makes a greater contribution to body weight ${ }^{(74)}$. High intakes of free sugars in excess of $20 \%$ total energy have been shown to increase VAT and ectopic fat in the liver, and produce marked effects on cardiometabolic risk factors ${ }^{(75)}$. Sugar-sweetened beverages have been identified as one of the main perpetrators for the delivery of such high intake of free sugars ${ }^{(76)}$, and thus potentially adverse metabolic effects, most notably in children and adolescents. However, the mean intake of free sugars in adult populations is well below this level of intake, and shows no significant association with the risk of CVD mortality until free sugars reach about $25 \%$ of total energy intake ${ }^{(77)}$. Soluble and insoluble dietary fibres from vegetable and cereal sources have been shown to exert favourable and pronounced effects on cardiometabolic risk factors ${ }^{(78)}$. While the underlying mechanisms for these beneficial effects of dietary fibre are not entirely clear, they may involve influences on the absorption of nutrients and gut microbiota that are independent of changes in body weight, and promotion of weight loss via the displacement of absorbable energy from dietary fat and carbohydrates.

\section{Dietary protein}

The impact of dietary protein on cardiometabolic risk may be mediated to a variable extent by its ability to counteract the adverse metabolic effects of sarcopenia, primarily by reducing anabolic resistance in skeletal muscle ${ }^{(79)}$. Since it is clear that the expression of cardiometabolic risk is enhanced in the presence of sarcopenia, a strategic priority must be to ensure an adequate intake of total protein, and then to consider the quality and time of ingestion of protein in relation to its anabolic effects on skeletal muscle ${ }^{(80)}$.

\section{Salt and alcohol}

The adverse consequences of salt and alcohol are largely centred on their effects in raising blood pressure. Accepted recommendations for the prevention and treatment of hypertension are to lower salt intake to achieve $1.5 \mathrm{~g} \mathrm{Na} / \mathrm{d}$ and moderation of alcohol intake (two units/d for men; one unit/d for women) ${ }^{(55)}$.

\section{Dietary patterns}

There is emerging evidence that the most effective dietary strategies for reducing risk and mortality from multifactorial CVD are based on the modification of multiple dietary components within a dietary pattern. Examples of such dietary patterns include variants of the Dietary Approaches to Stop Hypertension (DASH) $\operatorname{diet}^{(81,82)}$ (rich in fruits and vegetables (eight to ten servings/d), rich in low-fat dairy products (two to three servings/d), and a reduction in saturated fat and cholesterol and $\mathrm{K}$ intake $(120 \mathrm{mmol} / \mathrm{d}))$, and variants of the Mediterranean diet, as tested in the PREDIMED (Prevención con Dieta Mediterránea) study ${ }^{(83)}$. While these interventions were not specifically designed to lower cardiometabolic risk, it is reasonable to assume that since cardiometabolic risk has a multifactorial aetiology, it will be more responsive to multiple dietary changes within an altered dietary pattern. Focusing on whole foods and dietary patterns rather than single nutrients also reflects the way in which free-living humans consume nutrients and measure their dietary intake. This approach has been embraced recently by the new dietary guidelines in the Netherlands, which are based entirely on manipulating the intake of whole foods ${ }^{(84)}$.

The Mediterranean diet has become renowned as a dietary pattern associated with the prevention of CVD, which has been 
successfully tested in the PREDIMED study. The key principles of this dietary pattern are a largely plant-based diet, featuring fruit, vegetables, whole grains and nuts with moderate amounts of oily fish, olive oil and nuts, and smaller quantities of transfatty acids and SFA, red meat and processed and refined foods. The PREDIMED study intervened in men at increased risk of CVD with two variants of the Mediterranean diet supplemented with either extra-virgin olive oil (four tablespoons/d) or mixed nuts ( $\geq 3$ servings/week) against a control group who were advised to lower their intake of dietary fat. After 4.8 years, the oil- and nut-supplemented diets reduced the risk of cardiovascular events by 30 and $28 \%$, respectively, relative to the control.

\section{A personalised, phenotypic dietary approach for the treatment of androgen deprivation therapy-related cardiometabolic risk}

As previously described (Table 1), patients on ADT express a variable combination of drug-induced cardiometabolic risk factors. These can be subdivided into four risk categories or phenotypes, each of which will require different dietary priorities (Table 3).

Increased cardiometabolic risk can exist in the absence of a BMI in excess of $30 \mathrm{~kg} / \mathrm{m}^{2}$ (metabolic 'obesity'), but the concept of metabolic 'healthy' obesity has recently been challenged as a non-entity because of conflicting evidence for the presence of cardiometabolic risk in this condition ${ }^{(85)}$. This provides supporting rationale for placing all patients on ADT on a cardioprotective diet, which whilst having no negative effects on health, will be effective in the management of insulin resistance, dyslipidaemia, mild hypertension and vascular dysfunction. Superimposed on this is the priority to promote weight loss in overweight or obese patients, and the need to consider the preservation of muscle mass, if the patients shows signs of sarcopenia. The guidelines described in Table 4 were derived from the compilation of various dietary patterns which have been shown to reduce CVD risk.

\section{Management of weight and fat distribution}

Energy restriction is required to promote weight loss in ADT patients who are overweight or obese $(600 \mathrm{kcal}(2500 \mathrm{~kJ}) / \mathrm{d}$ reduction advised by the National Institute for Health and Care
Excellence (NICE)). While weight reduction alone appears to be achievable with a daily energy restriction, there is emerging evidence to suggest that additional improvements in insulin resistance and lean mass retention can be achieved through intermittent energy restriction ${ }^{(63,64)}$.

\section{Sarcopenia}

Limited studies have investigated skeletal muscle mass and metabolism in patients on ADT. It has been suggested that promyogenic drugs and myokines may be required to reduce the risk of sarcopenia and frailty in patients on $\mathrm{ADT}^{(86)}$. There is, as yet, no convincing evidence to support the efficacy of lifestyle interventions (nutrition and exercise interventions) as a means for attenuating muscle loss in patients on ADT. It is therefore reasonable to suggest that patients on ADT who show signs of muscle weakness follow similar therapeutic strategies to those who are at risk of muscle wastage, but not on ADT, such as regular protein intake and consumption of specific amino acids.

Weight-loss programmes tend to promote a loss of both fat mass and fat-free mass. A study of 275 men and women in the USA concluded that men decreasing their body weight by $10 \%$ should expect $35-40 \%$ of the weight loss to be fat-free mass ${ }^{(87)}$. To prevent this loss of muscle in overweight and obese patients with sarcopenia, many studies have examined how weight-loss programmes can be adapted to preserve lean tissue. Increasing protein intake to above $0.9 \mathrm{~g} / \mathrm{kg}$ per $\mathrm{d}$ was shown to be ineffective in preserving muscle mass in obese adults ${ }^{(88)}$. In contrast, a reduction in energy intake of $500 \mathrm{kcal}(2100 \mathrm{~kJ}) / \mathrm{d}$ with the consumption of whey-protein supplement $(20 \mathrm{~g}$ protein/d) was shown to promote a significant loss of fat mass whilst preserving fat-free mass, as compared with a control group in obese adults ${ }^{(89)}$. The synthesis of myofibrillar protein was also shown to be stimulated to a significantly greater extent in a weight-reducing diet that was supplemented with isolated whey protein $(1 \cdot 3 \pm 0 \cdot 1 \mathrm{~g}$ protein/kg per $\mathrm{d})$ as compared with soya or carbohydrate $^{(90)}$. Moreover, obese patients following a $600 \mathrm{kcal}$ $(2500 \mathrm{~kJ}) / \mathrm{d}$ energy reduction, resistance training programme and consuming a supplement containing whey protein, leucine and vitamin $\mathrm{D}$ containing $21 \mathrm{~g}$ protein (equating to an overall protein intake of $1 \cdot 11 \pm 0 \cdot 28 \mathrm{~g}$ protein $/ \mathrm{kg}$ per $\mathrm{d}$ ) preserved

Table 3. Cardiometabolic risk factors and dietary priorities in different physical phenotypes resulting from androgen deprivation therapy

\begin{tabular}{|c|c|c|c|c|}
\hline Phenotype & $\begin{array}{l}\text { Normal weight and no } \\
\text { sign of muscle wastage }\end{array}$ & $\begin{array}{l}\text { Normal weight and muscle } \\
\text { wastage }\end{array}$ & $\begin{array}{l}\text { Overweight and no sign of } \\
\text { muscle wastage }\end{array}$ & Overweight and muscle wastage \\
\hline $\begin{array}{l}\text { Cardiometabolic risks } \\
\text { factors }\end{array}$ & $\begin{array}{l}\text { Insulin resistance } \\
\text { Dyslipidaemia } \\
\text { Mild hypertension } \\
\text { Vascular dysfunction } \\
\text { Ectopic fat }\end{array}$ & $\begin{array}{l}\text { Insulin resistance } \\
\text { Dyslipidaemia } \\
\text { Mild hypertension } \\
\text { Vascular dysfunction } \\
\text { Ectopic fat } \\
\text { Sarcopenia }\end{array}$ & $\begin{array}{l}\text { Insulin resistance } \\
\text { Dyslipidaemia } \\
\text { Mild hypertension } \\
\text { Vascular dysfunction } \\
\text { Ectopic fat } \\
\text { Increased SAT leading to higher } \\
\quad \text { risk of cardiovascular events }\end{array}$ & $\begin{array}{l}\text { Insulin resistance } \\
\text { Dyslipidaemia } \\
\text { Mild hypertension } \\
\text { Vascular dysfunction } \\
\text { Ectopic fat } \\
\text { Sarcopenia } \\
\text { Increased SAT leading to higher } \\
\text { risk of cardiovascular events }\end{array}$ \\
\hline Dietary priorities & Prevention of CVD & $\begin{array}{l}\text { Prevention of CVD } \\
\text { Prevention of } \\
\text { muscle loss }\end{array}$ & $\begin{array}{l}\text { Prevention of CVD } \\
\text { Weight reduction }\end{array}$ & $\begin{array}{l}\text { Prevention of CVD } \\
\text { Prevention of muscle loss } \\
\text { Weight reduction }\end{array}$ \\
\hline
\end{tabular}

SAT, subcutaneous adipose tissue. 
Table 4. Dietary guidelines for the treatment of androgen deprivation therapy-related cardiometabolic risk

\begin{tabular}{|c|c|}
\hline Area & Guidelines \\
\hline Fruit and vegetables & $\begin{array}{l}\text { Five to ten portions of fruit and vegetables per d (all varieties such as fresh, frozen, canned) including } 10 \mathrm{~g} \text { of viscous fibres per } \\
1000 \mathrm{kcal}(4184 \mathrm{~kJ}) \text { from foods such as psyllium, okra and aubergine. Try to include tomato-based sauces containing onion, } \\
\text { garlic and olive oil at least twice per week }\end{array}$ \\
\hline High-protein foods & $\begin{array}{l}\text { As far as possible, try to split protein intake evenly throughout the day }(20-30 \mathrm{~g} \text { of high-quality protein is suggested per meal } \\
\text { containing } 2.5-2.8 \mathrm{~g} \text { leucine where possible). Plant-based protein such as legumes, nuts and seeds should each be } \\
\text { consumed at least three times per week. } 23 \mathrm{~g} \text { of almonds per } 1000 \mathrm{kcal}(4184 \mathrm{~kJ}) \text { consumed may lower cholesterol. Soya } \\
\text { protein should also be consumed regularly in similar quantities to almonds. Two eggs provide protein and essential amino } \\
\text { acids so should also be consumed regularly. Fish, especially oily fish, and seafood should be consumed at least three times } \\
\text { per week. White meat should be chosen over red meat where possible; however, red meat in quantities of less than } 490 \mathrm{~g} \text { per } \\
\text { week can still be part of a balanced diet providing it is lean and red and processed meat is not eaten on a daily basis }\end{array}$ \\
\hline Fats & $\begin{array}{l}\text { Fats should not contribute more than } 27 \% \text { of daily energy intake and saturated fat intake should be less than } 6 \% \text {. Saturated } \\
\text { fats such as butter, cream, lard and coconut oils should be limited. Spreadable fats should be used on average less than } \\
\text { once per d. Monounsaturated fats such as polyphenol-enriched olive oil should be included in the diet on a daily basis in } \\
\text { quantities of four or more tablespoons }\end{array}$ \\
\hline Starchy foods & $\begin{array}{l}\text { Starchy foods should be mainly whole grains and one should aim to include } 10 \mathrm{~g} \text { of viscous fibres from foods such as oats and } \\
\text { barley per } \mathrm{d}\end{array}$ \\
\hline Dairy products & $\begin{array}{l}\text { Dairy products should be restricted to low-fat varieties where possible and depending on weight should be consumed in } \\
\text { quantities of } 1.5-2.5 \text { servings per } d \text { if weight loss is a goal or } 2-3 \text { servings per } d \text { if weight maintenance is a goal }\end{array}$ \\
\hline $\begin{array}{l}\text { Functional foods and } \\
\text { vitamins }\end{array}$ & $\begin{array}{l}1 \mathrm{~g} \text { of plant sterols should be consumed for every } 1000 \mathrm{kcal}(4184 \mathrm{~kJ}) \\
\text { Foods containing vitamin D such as cod liver oil, salmon, sardines, etc. should also be included in the diet regularly }\end{array}$ \\
\hline Limited foods & $\begin{array}{l}\text { Commercial bakery goods and sweets should be limited and fat-free or low-fat should be chosen where possible } \\
\text { Sugar should also be swapped to sweetener when applicable } \\
\text { Soda drinks should be limited to the equivalent of less than one per d } \\
\text { Alcohol (for habitual drinkers only) should not exceed two drinks (preferably wine with meals) per } d \text { and alcohol-free days should } \\
\text { be observed } \\
\text { Caffeine consumption should be discussed with the general practitioner }\end{array}$ \\
\hline
\end{tabular}

appendicular muscle mass in comparison with a control group who did not consume a protein supplement ${ }^{(91)}$.

There is evidence to suggest that despite the similar effects of daily and intermittent energy restriction on weight loss, a higher percentage of lean mass may be preserved when undertaking intermittent energy restriction in the form of 'feeding' and 'fasting' days ${ }^{(63)}$

The timing of daily protein intake may also be important in this respect. The $24 \mathrm{~h}$ mixed muscle protein fractional synthesis rate was shown to be significantly higher when protein was evenly distributed throughout the day, as compared with skewing intake towards the evening meal ${ }^{(80)}$. A list of putative dietary factors with potential to prevent sarcopenia that results from advancing age includes protein, vitamin $\mathrm{D}$, antioxidants, long-chain PUFA and dietary patterns ${ }^{(92)}$.

\section{Psychological and clinical considerations}

As with any long-term diet and lifestyle modification, the ability for an individual to sustain change relies partly on the conscious and subconscious psychological strategies employed throughout the intervention. Michie et ll $^{(93)}$ have generated a framework of psychological techniques, used within the realms of diet and exercise regimens, and various behaviour change techniques to help individuals modify their behaviours. The latter includes, but is not limited to, goal setting, action planning, barrier identification and problem solving. Lifestyle interventions should not disregard the importance of considering behaviour change techniques which can greatly improve compliance and make an impact on outcomes. Abete et al. ${ }^{(61)}$ also highlight the relevance of investigating the intrinsic and extrinsic determining factors of dietary intake to aid compliance in promoting weight loss.
Dietitians and nutrition professionals often receive referrals for patients with cancer who require nutrition support for weight loss as a result of various cancer treatments. Men who are struggling with weight gain and increased cardiovascular risk may be overlooked because weight gain during cancer is perhaps not expected and not monitored in these patients. The holistic clinical care of a patient's health can also be diminished when individual elements of their condition are managed by different individuals. There is a need to highlight these issues in the service currently provided to patients with prostate cancer, and to generate guidelines that include referral criteria for various healthcare professionals who can advise on lifestyle changes that could reduce risk of CVD.

\section{Summary and conclusions}

ADT is highly effective in reducing mortality from prostate cancer, but certain forms of this therapy, most notably GnRH agonists, induce cardiometabolic abnormalities that can lead to premature cardiac events. While ADT-induced cardiometabolic risk is similar to that found in classic MetS, it is distinct in having a single underlying cause of testosterone deficiency, a more rapid onset, and being characterised by an accumulation of SAT, and sarcopenia and sarcopenic obesity.

Cardiometabolic risk can be reduced through energy restriction to promote weight loss, and by manipulating the composition of macronutrients, chiefly fat and carbohydrate, to target specific metabolic risk factors. Existing evidence for the beneficial role of diet in the prevention of ADT-related cardiometabolic risk is scant. Future treatment strategies should consider adopting an evidence-based approach with a personalised strategy that tailors dietary modification to the risk phenotype of the patient. Nutritional assessment should include consideration 
of the level to which a patient adheres to the Mediterranean diet, the quantity, quality and timing of protein intake, overall energy content and GI, while ensuring that World Cancer Research Fund guidelines are being met. We await the outcome of adequately powered RCT to establish if these dietary modifications will be successful in ameliorating ADT-related cardiometabolic risk.

\section{Acknowledgements}

Financial support was received from Movember and Prostate Cancer UK.

B. A. G., L. T., K. P. and S. F. planned, designed and performed literature searches for this review. B. A. G. and L. T. were the principal writers, and K. P. and S. F. provided peer review and editorial comments.

There are no conflicts of interest.

\section{References}

1. Prostate Cancer UK (2016) About prostate cancer. http:// www.cancerresearchuk.org/about-cancer/prostate-cancer (accessed July 2016).

2. Cancer Research UK (2016) Survival statistics for prostate cancer. http://www.cancerresearchuk.org/ health-professional/cancer-statistics/statistics-by-cancer-type/ prostate-cancer/survival (accessed July 2016)

3. Davis MK, Rajala JL, Tyldesley S, et al. (2015) The prevalence of cardiac risk factors in men with localized prostate cancer undergoing androgen deprivation therapy in British Columbia, Canada. J Oncol 2015, 820403.

4. O'Farrell S, Garmo H, Holmberg L, et al. (2015) Risk and timing of cardiovascular disease after androgen-deprivation therapy in men with prostate cancer. J Clin Oncol 33, 1243-1251.

5. Morote J, Gómez-Caamaño A, Alvarez-Ossorio JL, et al. (2015) The metabolic syndrome and its components in patients with prostate cancer on androgen deprivation therapy. J Urol 193, 1963-1969.

6. Jespersen CG, Nørgaard M \& Borre M (2014) Androgendeprivation therapy in treatment of prostate cancer and risk of myocardial infarction and stroke: a nationwide Danish population-based cohort study. Eur Urol 65, 704-709.

7. Teoh JY, Chan SY, Chiu PK, et al. (2015) Risk of acute myocardial infarction after androgen-deprivation therapy for prostate cancer in a Chinese population. BJU Int 116, 382-387.

8. Gandaglia G, Sun M, Popa I, et al. (2015) Cardiovascular mortality in patients with metastatic prostate cancer exposed to androgen deprivation therapy: a populationbased study. Clin Genitourin Cancer 13, e123-e130.

9. Levine GN, D'Amico AV, Berger P, et al. (2010) Androgendeprivation therapy in prostate cancer and cardiovascular risk: a science advisory from the American Heart Association, American Cancer Society, and American Urological Association: endorsed by the American Society for Radiation Oncology. Circulation 121, 833-840.

10. Keating NL, O'Malley AJ \& Smith MR (2006) Diabetes and cardiovascular disease during androgen deprivation therapy for prostate cancer. J Clin Oncol 24, 4448-4456.

11. Tsai HK, D'Amico AV, Sadetsky N, et al. (2007) Androgen deprivation therapy for localized prostate cancer and the risk of cardiovascular mortality. I Natl Cancer Inst 99, 1516-1524.
12. Bosco C, Bosnyak Z, Malmberg A, et al. (2015) Quantifying observational evidence for risk of fatal and nonfatal cardiovascular disease following androgen deprivation therapy for prostate cancer: a meta-analysis. Eur Urol $\mathbf{6 8}$, 386-396.

13. Smith JC, Bennett S, Evans LM, et al. (2001) The effects of induced hypogonadism on arterial stiffness, body composition, and metabolic parameters in males with prostate cancer. J Clin Endocrinol Metab 86, 4261-4267.

14. Gilbert SE, Tew GA, Bourke L, et al. (2013) Assessment of endothelial dysfunction by flow-mediated dilatation in men on long-term androgen deprivation therapy for prostate cancer. Exp Physiol 98, 1401-1410.

15. Oka R, Utsumi T, Endo T, et al. (2016) Effect of androgen deprivation therapy on arterial stiffness and serum lipid profile changes in patients with prostate cancer: a prospective study of initial 6-month follow-up. Int J Clin Oncol 21, 389-396.

16. Knutsson A, Hsiung S, Celik S, et al. (2016) Treatment with a GnRH receptor agonist, but not the GnRH receptor antagonist degarelix, induces atherosclerotic plaque instability in $\mathrm{ApoE}^{-/-}$mice. Sci Rep 6, 26220.

17. Roach M, Bae K, Speight J, et al. (2008) Short-term neoadjuvant androgen deprivation therapy and externalbeam radiotherapy for locally advanced prostate cancer: long-term results of RTOG 8610. J Clin Oncol 26, 585-591.

18. Alibhai SM, Duong-Hua M, Sutradhar R, et al. (2009) Impact of androgen deprivation therapy on cardiovascular disease and diabetes. J Clin Oncol 27, 3452-3458.

19. Nguyen PL, Je Y, Schutz FA, et al. (2011) Association of androgen deprivation therapy with cardiovascular death in patients with prostate cancer: a meta-analysis of randomized trials. JAMA 306, 2359-2366.

20. Nguyen PL, Chen MH, Beckman JA, et al. (2012) Influence of androgen deprivation therapy on all-cause mortality in men with high-risk prostate cancer and a history of congestive heart failure or myocardial infarction. Int $J$ Radiat Oncol Biol Phys 82, 1411-1416.

21. Voog JC, Paulus R, Shipley WU, et al. (2016) Cardiovascular mortality following short-term androgen deprivation in clinically localized prostate cancer: an analysis of RTOG 94-08. Eur Urol 69, 204-210.

22. Albertsen PC, Klotz L, Tombal B, et al. (2014) Cardiovascular morbidity associated with gonadotropin releasing hormone agonists and an antagonist. Eur Urol 65, 565-573.

23. Ziehr DR, Chen MH, Zhang D, et al. (2015) Association of androgen-deprivation therapy with excess cardiac-specific mortality in men with prostate cancer. BJU Int 116, 358-365.

24. Monzó-Gardiner JI \& Herranz-Amo F (2015) Cardiovascular mortality in patients with prostate cancer exposed to androgen deprivation therapy. Actas Urol Esp 39, 518-522.

25. Gandaglia G, Sun M, Popa I, et al. (2014) The impact of androgen-deprivation therapy (ADT) on the risk of cardiovascular (CV) events in patients with non-metastatic prostate cancer: a population-based study. BJU Int 114, E82-E89.

26. Rutter MK, Meigs JB, Sullivan LM, et al. (2005) Insulin resistance, the metabolic syndrome, and incident cardiovascular events in the Framingham Offspring Study. Diabetes 54, 3252-3257.

27. Lohmann AE, Goodwin PJ, Chlebowski RT, et al. (2016) Association of obesity-related metabolic disruptions with cancer risk and outcomes. J Clin Oncol 34, 4249-4255.

28. Hopkins BD, Gonclaves MD \& Cantley LC (2016) Obesity and cancer mechanisms:cancer metabolism. J Clin Oncol 34, 4277-4283. 
29. de Haas EC, Oosting SF, Lefrandt JD, et al. (2010) The metabolic syndrome in cancer survivors. Lancet Oncol 11, 193-203.

30. Kahn R (2008) Metabolic syndrome - what is the clinical usefulness? Lancet 371, 1892-1893.

31. Jackson KG, Walden CM, Murray P, et al. (2012) A sequential two meal challenge reveals abnormalities in postprandial TAG but not glucose in men with increasing numbers of metabolic syndrome components. Atherosclerosis 220, 237-243.

32. Wahi G \& Anand SS (2013) Race/ethnicity, obesity, and related cardio-metabolic risk factors: a life-course perspective. Curr Cardiovasc Risk Rep 7, 326-335.

33. Ouchi N, Parker JL, Lugus JJ, et al. (2011) Adipokines in inflammation and metabolic disease. Nat Rev Immunol 11, 85-97.

34. Sattar N \& Gill JM (2014) Type 2 diabetes as a disease of ectopic fat? BMC Med 12, 123.

35. Nguyen PL, Jarolim P, Basaria S, et al. (2015) Androgen deprivation therapy reversibly increases endotheliumdependent vasodilation in men with prostate cancer. J Am Heart Assoc 4, e001914.

36. Grossmann M \& Wittert G (2012) Androgens, diabetes and prostate cancer. Endocr Relat Cancer 19, F47-F62.

37. Smith MR, Saad F, Egerdie B, et al. (2012) Sarcopenia during androgen-deprivation therapy for prostate cancer. J Clin Oncol 30, 3271-3276.

38. Saylor PJ \& Smith MR (2009) Metabolic complications of androgen deprivation therapy for prostate cancer. $J$ Urol 189, S34-S42; discussion S43-S44.

39. Efstathiou JA, Bae K, Shipley WU, et al. (2009) Cardiovascular mortality after androgen deprivation therapy for locally advanced prostate cancer: RTOG 85-31. J Clin Oncol 27, 92-99.

40. Punnen S, Cooperberg MR, Sadetsky N, et al. (2011) Androgen deprivation therapy and cardiovascular risk. J Clin Oncol 29, 3510-3516.

41. Wall BA, Galvão DA, Fatehee N, et al. (2015) Reduced cardiovascular capacity and resting metabolic rate in men with prostate cancer undergoing androgen deprivation: a comprehensive cross-sectional investigation. Adv Urol 2015, 976235 .

42. Srikanthan P, Hevener AL \& Karlamangla AS (2010) Sarcopenia exacerbates obesity-associated insulin resistance and dysglycemia: findings from the National Health and Nutrition Examination Survey III. PLOS ONE 5, e10805.

43. Lee J, Hong YP, Shin HJ, et al. (2016) Associations of sarcopenia and sarcopenic obesity with metabolic syndrome considering both muscle mass and muscle strength. J Prev Med Public Health 49, 35-44.

44. Cheung AS, Zajac JD \& Grossmann M (2014) Muscle and bone effects of androgen deprivation therapy: current and emerging therapies. Endocr Relat Cancer 21, R371-R394.

45. Ma J, Hwang SJ, McMahon GM, et al. (2016) Mid-adulthood cardiometabolic risk factor profiles of sarcopenic obesity. Obesity (Silver Spring) 24, 526-534.

46. Loomba R \& Sanyal AJ (2013) The global NAFLD epidemic. Nat Rev Gastroenterol Hepatol 10, 686-690.

47. Kotronen A \& Yki-Järvinen H (2008) Fatty liver: a novel component of the metabolic syndrome. Arterioscler Thromb Vasc Biol 28, 27-38.

48. Preiss D \& Sattar N (2007) Metabolic syndrome, dysglycaemia and vascular disease: making sense of the evidence. Heart 93, 1493-1496.

49. Kelishadi R, Cook SR, Adibi A, et al. (2009) Association of the components of the metabolic syndrome with non-alcoholic fatty liver disease among normal-weight, overweight and obese children and adolescents. Diabetol Metab Syndr 1, 29.

50. Conti CR (2002) Evolution of NCEP guidelines: ATP1ATPIII risk estimation for coronary heart disease in 2002. National Cholesterol Education Program. Clin Cardiol 25, 89-90.

51. Jenkins DJ, Kendall CW, Faulkner DA, et al. (2006) Assessment of the longer-term effects of a dietary portfolio of cholesterol-lowering foods in hypercholesterolemia. Am J Clin Nutr 83, 582-591.

52. Jenkins DJ, Jones PJ, Lamarche B, et al. (2011) Effect of a dietary portfolio of cholesterol-lowering foods given at 2 levels of intensity of dietary advice on serum lipids in hyperlipidemia: a randomized controlled trial. JAMA $\mathbf{3 0 6}$, 831-839.

53. Muzio F, Mondazzi L, Harris WS, et al. (2007) Effects of moderate variations in the macronutrient content of the diet on cardiovascular disease risk factors in obese patients with the metabolic syndrome. Am J Clin Nutr 86, 946-951.

54. Laddu D, Dow C, Hingle M, et al. (2011) A review of evidence-based strategies to treat obesity in adults. Nutr Clin Pract 26, 512-525.

55. Appel LJ, Brands MW, Daniels SR, et al. (2006) Dietary approaches to prevent and treat hypertension: a scientific statement from the American Heart Association. Hypertension 47, 296-308.

56. Arab L, Su J, Steck SE, et al. (2013) Adherence to World Cancer Research Fund/American Institute for Cancer Research lifestyle recommendations reduces prostate cancer aggressiveness among African and Caucasian Americans. Nutr Cancer 65, 633-643.

57. NICE (2016) Obesity: identification, assessment and management: 1.7 Dietary. https://www.nice.org.uk/guidance/ cg189 (accessed July 2016).

58. Garaulet M, Gómez-Abellán P, Alburquerque-Béjar JJ, et al. (2013) Timing of food intake predicts weight loss effectiveness. Int J Obes (Lond) 37, 604-611.

59. Knutson KL (2012) Does inadequate sleep play a role in vulnerability to obesity? Am J Hum Biol 24, 361-371.

60. Harrington DM, Martin CK, Ravussin E, et al. (2013) Activity related energy expenditure, appetite and energy intake: potential implications for weight management. Appetite $\mathbf{6 7}$, $1-7$.

61. Abete I, Astrup A, Martínez JA, et al. (2010) Obesity and the metabolic syndrome: role of different dietary macronutrient distribution patterns and specific nutritional components on weight loss and maintenance. Nutr Rev 68, 214-231.

62. Mattson MP, Allison DB, Fontana L, et al. (2014) Meal frequency and timing in health and disease. Proc Natl Acad Sci U S A 111, 16647-16653.

63. Varady KA (2011) Intermittent versus daily calorie restriction: which diet regimen is more effective for weight loss? Obes Rev 12, e593-e601.

64. Harvie MN, Pegington M, Mattson MP, et al. (2011) The effects of intermittent or continuous energy restriction on weight loss and metabolic disease risk markers: a randomized trial in young overweight women. Int J Obes (Lond) 35, 714-727.

65. Klempel MC, Kroeger CM, Bhutani S, et al. (2012) Intermittent fasting combined with calorie restriction is effective for weight loss and cardio-protection in obese women. Nutr J 11, 98.

66. Smith U (2015) Abdominal obesity: a marker of ectopic fat accumulation. J Clin Invest 125, 1790-1792. 
67. Rosqvist F, Iggman D, Kullberg J, et al. (2014) Overfeeding polyunsaturated and saturated fat causes distinct effects on liver and visceral fat accumulation in humans. Diabetes $\mathbf{6 3}$, 2356-2368.

68. Jebb SA, Lovegrove JA, Griffin BA, et al. (2010) Effect of changing the amount and type of fat and carbohydrate on insulin sensitivity and cardiovascular risk: the RISCK (Reading, Imperial, Surrey, Cambridge, and Kings) trial. Am J Clin Nutr 92, 748-758.

69. Jump DB, Depner CM \& Tripathy S (2012) Omega-3 fatty acid supplementation and cardiovascular disease. J Lipid Res 53, 2525-2545.

70. Araya J, Rodrigo R, Videla LA, et al. (2004) Increase in longchain polyunsaturated fatty acid $n-6 / n-3$ ratio in relation to hepatic steatosis in patients with non-alcoholic fatty liver disease. Clin Sci (Lond) 106, 635-643.

71. Scorletti E, Bhatia L, McCormick KG, et al. (2014) Effects of purified eicosapentaenoic and docosahexaenoic acids in nonalcoholic fatty liver disease: results from the WELCOME* study. Hepatology 60, 1211-1221.

72. Cocate PG, Pereira LG, Marins JC, et al. (2011) Metabolic responses to high glycemic index and low glycemic index meals: a controlled crossover clinical trial. Nutr J 10, 1 .

73. Te Morenga L, Mallard S \& Mann J (2012) Dietary sugars and body weight: systematic review and meta-analyses of randomised controlled trials and cohort studies. BMJ $\mathbf{3 4 6}$, e7492.

74. Anderson JJ, Celis-Morales CA, Mackay DF, et al. (2016) Adiposity among 132479 UK Biobank participants; contribution of sugar intake vs other macronutrients. Int $J$ Epidemiol (epublication ahead of print version 12 July 2016)

75. Stanhope KL, Schwarz JM, Keim NL, et al. (2009) Consuming fructose-sweetened, not glucose-sweetened, beverages increases visceral adiposity and lipids and decreases insulin sensitivity in overweight/obese humans. J Clin Invest 119, 1322-1334.

76. Scientific Advisory Committee on Nutrition (SACN) (2015) Carbohydrates and Health. London: TSO (The Stationery Office).

77. Yang Q, Zhang Z, Gregg EW, et al. (2014) Added sugar intake and cardiovascular diseases mortality among US adults. JAMA Intern Med 174, 516-524.

78. Riccioni G, Sblendorio V, Gemello E, et al. (2012) Dietary fibers and cardiometabolic diseases. Int J Mol Sci 13, 1524-1540.

79. Nowson C \& O'Connell S (2015) Protein requirements and recommendations for older people: a review. Nutrients $\mathbf{7}$, 6874-6899.

80. Mamerow MM, Mettler JA, English KL, et al. (2014) Dietary protein distribution positively influences 24-h muscle protein synthesis in healthy adults. J Nutr 144, 876-880.

81. Moore TJ, Conlin PR, Ard J, et al. (2001) DASH (Dietary Approaches to Stop Hypertension) diet is effective treatment for stage 1 isolated systolic hypertension. Hypertension 38, 155-158

82. Blumenthal JA, Babyak MA, Hinderliter A, et al. (2010) Effects of the DASH diet alone and in combination with exercise and weight loss on blood pressure and cardiovascular biomarkers in men and women with high blood pressure: the ENCORE study. Arch Intern Med 170, 126-135.

83. Estruch R, Ros E, Salas-Salvadó J, et al. (2013) Primary prevention of cardiovascular disease with a Mediterranean diet. $N$ Engl J Med 368, 1279-1290.

84. Kromhout D, Spaaij CJ, de Goede J, et al. (2016) The 2015 Dutch food-based dietary guidelines. Eur J Clin Nutr 70, 869-878.
85. Fan J, Song Y, Chen Y, et al. (2013) Combined effect of obesity and cardio-metabolic abnormality on the risk of cardiovascular disease: a meta-analysis of prospective cohort studies. Int J Cardiol 168, 4761-4768.

86. Basaria S \& Bhasin S (2012) Targeting the skeletal musclemetabolism axis in prostate-cancer therapy. $N$ Engl $J$ Med 367, 965-967.

87. Dixon JB, Lambert EA, Grima M, et al. (2015) Fat-free mass loss generated with weight loss in overweight and obese adults: what may we expect? Diabetes Obes Metab 17, 91-93.

88. Backx EM, Tieland M, Borgonjen-van den Berg KJ, et al. (2016) Protein intake and lean body mass preservation during energy intake restriction in overweight older adults. Int J Obes (Lond) 40, 299-304.

89. Frestedt JL, Zenk JL, Kuskowski MA, et al. (2008) A wheyprotein supplement increases fat loss and spares lean muscle in obese subjects: a randomized human clinical study. Nutr Metab (Lond) 5, 8.

90. Hector AJ, Marcotte GR, Churchward-Venne TA, et al. (2015) Whey protein supplementation preserves postprandial myofibrillar protein synthesis during short-term energy restriction in overweight and obese adults. $J$ Nutr $\mathbf{1 4 5}$, 246-252.

91. Verreijen AM, Verlaan S, Engberink MF, et al. (2015) A high whey protein-, leucine-, and vitamin D-enriched supplement preserves muscle mass during intentional weight loss in obese older adults: a double-blind randomized controlled trial. Am J Clin Nutr 101, 279-286.

92. Robinson S, Cooper C \& Aihie Sayer A (2012) Nutrition and sarcopenia: a review of the evidence and implications for preventive strategies. J Aging Res 2012, 510801.

93. Michie S, Ashford S, Sniehotta FF, et al. (2011) A refined taxonomy of behaviour change techniques to help people change their physical activity and healthy eating behaviours: the CALO-RE taxonomy. Psychol Health 26, 1479-1498.

94. Nobes JP, Langley SE, Klopper T, et al. (2012) A prospective, randomized pilot study evaluating the effects of metformin and lifestyle intervention on patients with prostate cancer receiving androgen deprivation therapy. BJU Int 109, 1495-1502.

95. Bourke L, Gilbert S, Hooper R, et al. (2014) Lifestyle changes for improving disease-specific quality of life in sedentary men on long-term androgen-deprivation therapy for advanced prostate cancer: a randomised controlled trial. Eur Urol 65, 865-872.

96. D'Amico AV, Chen MH, Renshaw AA, et al. (2008) Androgen suppression and radiation vs radiation alone for prostate cancer: a randomized trial. JAMA 299, 289-295.

97. Messing EM, Manola J, Yao J, et al. (2006) Immediate versus deferred androgen deprivation treatment in patients with node-positive prostate cancer after radical prostatectomy and pelvic lymphadenectomy. Lancet Oncol 7, 472-479.

98. Bolla M, Van Tienhoven G, Warde P, et al. (2010) External irradiation with or without long-term androgen suppression for prostate cancer with high metastatic risk: 10-year results of an EORTC randomised study. Lancet Oncol 11, 1066-1073.

99. Schröder FH, Kurth K-H, Fossa SD, et al. (2009) Early versus delayed endocrine treatment of T2-T3 pN1-3 M0 prostate cancer without local treatment of the primary tumour: final results of European Organisation for the Research and Treatment of Cancer protocol 30846 after 13 years of follow-up (a randomised controlled trial). Eur Urol 55, 14-22. 
100. Studer UE, Whelan P, Albrecht W, et al. (2006) Immediate or deferred androgen deprivation for patients with prostate cancer not suitable for local treatment with curative intent: European Organisation for Research and Treatment of Cancer (EORTC) trial 30891. J Clin Oncol 24, 1868-1876.

101. Denham JW, Steigler A, Lamb DS, et al. (2011) Short term neoadjuvant androgen deprivation and radiotherapy for locally advanced prostate cancer: 10 -year data from the TROG 96.01 randomised trial. Lancet Oncol 12, 451-459.

102. Azoulay L, Yin H, Benayoun S, et al. (2011) Androgendeprivation therapy and the risk of stroke in patients with prostate cancer. Eur Urol 60, 1244-1250.

103. Hu JC, Williams SB, O'Malley AJ, et al. (2012) Androgendeprivation therapy for nonmetastatic prostate cancer is associated with an increased risk of peripheral arterial disease and venous thromboembolism. Eur Urol 61, 1119-1128.
104. Keating NL, O'Malley AJ, Freedland SJ, et al. (2010) Diabetes and cardiovascular disease during androgen deprivation therapy: observational study of veterans with prostate cancer. J Natl Cancer Inst 102, 39-46.

105. Martín-Merino E, Johansson S, Morris T, et al. (2011) Androgen deprivation therapy and the risk of coronary heart disease and heart failure in patients with prostate cancer: a nested case-control study in UK primary care. Drug Saf 34, 1061-1077.

106. Van Hemelrijck M, Adolfsson J, Garmo H, et al. (2010) Risk of thromboembolic diseases in men with prostate cancer: results from the population-based PCBaSe Sweden. Lancet Oncol 11, 450-458.

107. Van Hemelrijck M, Garmo H, Holmberg L, et al. (2010) Absolute and relative risk of cardiovascular disease in men with prostate cancer: results from the population-based PCBaSe Sweden. J Clin Oncol 28, 3448-3456. 